\title{
AUGMENTATION THE LIFETIME OF THE OPTIMAL COOPERATIVE SPECTRUM SENSING SENSOR NETWORK
}

\author{
Hayder S. Rashid Hujijo ${ }^{1}$ and Mohammed J. Alhasan ${ }^{2}$ \\ ${ }^{1,2}$ Department of Communication Techniques Engineering, Engineering Technical \\ College/Najaf, Al-Furat Al-Awsat Technical University, Najaf, Iraq \\ ${ }^{1}$ h.s.rashid@atu.edu.iq, ${ }^{2}$ m.j.alhasan@atu.edu.iq
}

\begin{abstract}
In a sensor-aided cognitive radio network, collaborating batterypowered sensors are deployed to aid the network in cooperative spectrum sensing. These sensors consume energy for spectrum sensing and therefore deplete their lifetime. Therefore, a study is required to minimize the sensing energy consumed and maximize the lifetime, in order to satisfy a larger spectrum utilization. However, this study focused on the spectrum sensing as a major factor at each cognitive radio network. A new cooperative sensing scheme established on the energy detection of each individual sensor was proposed and evaluated in this work by mean of limitation the energy of the wireless sensor elements. The main goals behind the new design are to minimize the sensing energy of each sensor and accurately select appropriate participant sensors in each cooperative sensing operation process. Lastly, the numerical results show that according to the unprecedented mathematical model, which proposed in this study, the lifetime of the cognitive network is increased, as well as, the application of available spectrum by secondary devices become more efficient compared with the existing algorithms. In general, this modification leads to improve the target detection performance.
\end{abstract}

Keywords - Wireless sensor networks, Cognitive radio, Spectrum sensing, Lifetime, Average sensing energy

\section{INTRODUCTION}

Cognitive Radio (CR), proposed by J. Mitola in [1], has arisen as the innovative dynamic spectrum access technology [2] to enhance the existing assigned spectrum. Depending on the Federal Communications Commission (FCC) [3], the actually using spectrum is only varying from $15 \%$ to $85 \%$ of its value and it completely relying on temporal variations and geographical. According to the fundamental of a cognitive radio network, the unlicensed secondary users (SUs) will be able to utilize the available licensed spectrum when it is unused by the licensed primary users (PUs) [4]. Nonetheless, for the reason of avoiding the interfering with the primary transmission, the occupied spectrum must be vacated as soon as possible when the primary users starts utilizing it. Therefore, spectrum sensing detection is fixed as a compulsory feature within the IEEE P802.22 standard [5], in order to make a CR device capable to identify and adapt to the primary usage of a spectrum band. According to the IEEE P802.22 standard, the sensing performance metric is summarized in terms of sensing receiver sensitivity, channel detection time, detection probability, and false alarm probability. However, investigating,

Received: July 20, 2019

Reviewed: January 15, 2020

Accepted: January 17, 2020

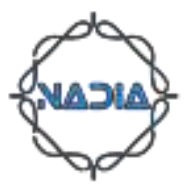


analyzing, and enhancing the sensing performance have stood out as one of the most important issues in spectrum sensing [6,7].

Several previous studies investigate the cooperative spectrum sensing networks in terms of improving its performance. For instance, the superior of the performance of the energy detection (ED) for Additive white Gaussian noise (AWGN) channels than the performance of the Rayleigh fading channels for lower threshold has been proved in [8]. The authors optimize the threshold for different voting rules and different Pf values. Thus, this investigation study has led to improve the sensing capability of the entire system. While an adaptive threshold ED scheme which is a function of conventional threshold and signal-to-noise ratio (SNR) of the primary device signal (PU) at Cognitive Radio was described in the study [9]. The study concluded that the probability of detection for their scheme under cooperative and non-cooperative spectrum sensing was clearly increased.

On the other hand, the most effective normalized ED based cooperative sensing scheme was proposed in [10]. The new normalizing method leads to highlight the fundamental of log likelihood ratio test and consider the reporting errors at the fusion center. In addition, a simplified likelihood ratio test was proposed in the same study, in order to simplify the decision making and threshold setting. The designed scheme effected on dropping the prerequisite of prior information on SNR and the variance of reporting errors.

The challenges of the energy minimization's problems in cooperative CR were faced in the study [11]. The researchers formulate the optimization problem based on the constraints of detection accuracy, in order to develop the optimal sensing time and the number of sensors, which is lead to minimize the energy consumption. In addition, the researchers in [12] proposed an optimization for cooperative spectrum sensing, to maximize the efficiency of spectrum in the interference limited primary system. In order to increase the efficiency of spectrum sensing.

Moreover, the main problem behind maximizing the transmission duration's ratio over the entire sensing cycle was briefly highlighted in the previous study [13]. While the researchers in [14] focused on how to minimize the used energy in distributed sensing. Accordingly, the study reached considerable save the energy consumed by presenting a new method based on combined sleeping and censoring scheme. The proposed scheme results conclude that the new method has the ability to ensure only transmitting informative decisions to the fusion center.

In term of the lifetime considerations, the lifetime of each node regarding the energy constrained of the wireless sensor networks was improved in [15]. The study presents a sub-fusion center among the sensors and the main fusion center. The sub-fusion center integrates the sensors' local decisions and then sends an integrated decision to the main fusion center. This improving affected increasing the nodes' lifetime. It is worth mentioning that, recently, the researches go towered the Internet of Things (IoT) research area, in order to combine the wireless sensor networks and social networks in a completely single system [16], [17]. The main aim of this combining is to promote developed embedded systems.

The current non-cooperative spectrum sensing technology has difficulty meeting the application requirements of CWSN at present [18]. Although NCSS has the benefits of low computation and simple implementation, however, it typically cannot take the correct decision on the PU status due to the terrible of communication environment [19]. Recent work has shown that cooperative spectrum sensing can effectively improve the sensing performance of CWSN, therefore, the main goal of this study is to present an unprecedented cooperative sensing scheme for energy detection related to the cognitive radio wireless sensor network. Firstly, the proposed work considers that the ED spectrum sensing can individually work without requiring any information from the PUs. Secondly, the study aims to maximize the lifetime of the cognitive radio wireless sensor (CRWS) 
network. The suggested scheme works on increasing the network's lifetime by mean of minimizing the sensing energy of each separate sensor and carefully selecting suitable participant sensors. The proposed selection aims to maximize the utilization of the cooperative sensing process in the team of the detection accuracy.

This article is organized as follows: the motivation and the related works described in Section 1. While the system mathematical model was driven in Section 2. Next, the suggested algorithm is clarified in Sections 3 and 4. The simulation results are described and briefly discussed in Section 5, and lastly, conclusions and future direction are specified in the last Section 6.

\section{NUMERICAL MODEL}

The activity of the PU on a proposed spectrum band can be detected according to the sensors network $(\mathcal{N})$. In fact, the instant SNR, which calls signal-to-noise-ratio $\left(Y_{i}\right)$, for each individual sensor $\left({ }^{i}\right)$ within the sensor network changes from sensor to the next sensor according to the communication environment of the surrounding wireless. Therefore, a single cognitive radio node and single of fusion center (FC) are proposed to present the CR sensor network in this study. However, each individual sensor node inside the entire network has own total energy, which can be denoted by $\left(\varepsilon_{T i}\right)$.

However, the tail probability of the Gaussian distribution can be calculated in terms of the Gaussian Q-function [20] as:

$$
Q(z)=\frac{1}{\sqrt{2 \pi}} \int_{z}^{m e} e^{-\frac{x^{2}}{2} d x}
$$

Based on the mathematical model that driven in the previous work [11], [12], the real time values of the single-node right detection and false alarm probabilities $P_{d \mathrm{di}}$ and $P_{f \mathrm{i}}$, respectively, can be easily determined as follows:

$$
\begin{aligned}
& P_{d i}=Q(z)\left(\frac{\lambda-2 t_{s i} W\left(Y_{i}+1\right) \sigma_{n}^{2}}{2 \sqrt{t_{s i} W}\left(\gamma_{i}+1\right) \sigma_{n}^{2}}\right) \\
& P_{f i}=Q(z)\left(\frac{\lambda-2 t_{s i} W \sigma_{n}^{2}}{2 \sqrt{t_{s i} W} \sigma_{n}^{2}}\right)
\end{aligned}
$$

Where, ${ }^{\lambda}$ is the energy threshold used by the energy detector, ${ }^{2} t_{g i} W$ is the number of samples, $t_{g i}$ is the spectrum sensing interval, ${ }^{W}$ is the spectrum bandwidth, and $\sigma_{n \mathrm{n}}$ is the ground noise.

Indeed, it is possible to model the traffic pattern of PU into two phases; independent and identically distributed (i.i.d) $\mathrm{ON}-\mathrm{OFF}$ random process [17], whose $\mathrm{ON}$ and $\mathrm{OFF}$ periods are exponentially distributed with the means in terms of time as $T_{\text {on }}$ and $T_{\text {off }}$, respectively. However, the $P_{d i}$ and $P_{f i}$ of each sensor node $\left({ }^{i}\right)$ can be represented [21], as:

$$
\begin{aligned}
& \hat{P}_{d i}=P a_{o n n} \cdot P_{d i}=\frac{T_{o n}}{T_{o n}+T_{o f f}} \cdot P_{d i} \\
& \hat{P}_{f i}=P a_{o f f} \cdot P_{f i}=\frac{T_{o f f}}{T_{o f f}+T_{o n}} \cdot P_{f i}
\end{aligned}
$$

Depending on Cognitive Radio of individual sensor node $\left({ }^{i}\right)$ and for simplifying the model the primary parameter $\left(\xi_{i}\right)$ was assumed. The main goal behind this assumption is 
to indicate whether the sensor node $\left(^{(i)}\right.$ is involved in the process or not, i.e. in term of the mathematical description, the primary parameter $\left(\xi_{i}\right)$ can be present as:

$\xi_{\mathrm{i}}=\left\{\begin{array}{l}0, \text { the sensor node }(i) \text { is not involved } \\ 1, \text { the sensor node }(i) \text { is involved }\end{array}\right.$

Based on the fusion center performs the "OR-rule" [12], [22] and the binary parameter

$\left(\xi_{i}\right)$, the final cooperative detection and false alarm probabilities $Q_{d}$ and $Q_{f}$ from aggregating the single-node detection and false alarm probabilities can be represented as follow:

$Q_{d}=1-\prod_{i=1}^{n} \xi_{i}\left(1-\hat{P}_{d i}\right)$

$Q_{f}=1-\prod_{i=1}^{n} \xi_{i}\left(1-\hat{P}_{f i}\right)$

\section{THE SENSING TIME SCHEME}

The main goal of this calculation scheme is to set the spectrum sensing time at any sensor node $\left({ }^{i}\right)$. However, assuming that cost function at each sensor node $\left(C F_{i}\right)$ can be driven by dividing the right detection probability $\left(\widehat{P}_{d i}\right)$, which is presented in question 4 , over the energy consumed of the same sensing node $\left(E C_{i}\right)$, which is approximately equal to multiply the sensing energy $\left(e_{s}\right)$ with its spectrum sensing interval for the same sensing node $\left(t_{g i}\right)$. Accordingly, the cost function of the senor node can be calculated by depending on the above assumption and equations 2 and 4, as follow:

$C F_{\mathrm{i}}=\frac{P_{d i}}{E C_{\mathrm{i}}}$

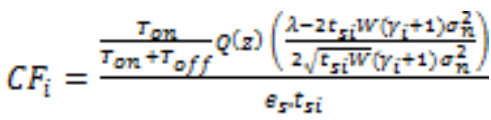

Where, the values of $T_{\text {on }}, T_{\text {off }}$, and $e_{g}$ are constant. Thus, it's possible to collect it in one constant $\left({ }^{T e}\right)$ to simplify the mathematical model, as follow:

$T e=\frac{T_{o n}}{\left(T_{o n}+T_{o f f}\right) e_{x}}$

In this case, the final cost function $\left(F C F_{i}\right)$ per each sensing node will calculate basing on equations 10 and 11, as follow:

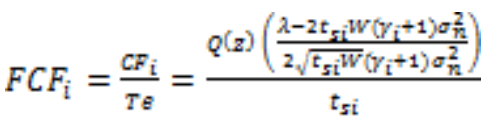

Thus, the optimal sensing interval $\left(t_{s i}^{*}\right)$ that leads to minimize the total sensing energy consumed by the cooperative spectrum sensing can be solved by the following maximization problem [11]:

$t_{g i}^{*}=\arg \left[\max \left(F C F_{i}\right)\right]$

Finding out an analytical solving to the optimization equation (13) is hard to achieve in high accuracy rate. While several approximation attempts were presented in the previous related works, the solving presented in a study [23] seems to have more acceptability with solving approach accuracy reached to $80 \%$ of the exact result of the original optimization. Therefore, the formula (13) can be represented in polynomial time when $\mathrm{z}$ is positive as follow: 
$t_{a i}^{*}=\frac{1}{W}\left[\sqrt{\frac{x^{2}}{4\left(Y_{i}+1\right)}+1}+1\right]$

The final analytical solving has completely depended on the value of the independent variable z. Thus the above equations will be solved in three approximation approaches as follow [11]:

a) When $-0.5 \leq z \leq 0.5$ (Linearization case)

It's can be clearly noted from the Gaussian Q-function (1) that the solution of the exponential equation can be present in a liner form when $\mathrm{z}$ varies from -0.5 to 0.5 . However, the following linearization is described to accurately and analytically solve the optimization equation (13).

$t_{g i}^{*}=\frac{1}{W}\left[-\pi \sqrt{1+\frac{\mathrm{a} \lambda}{\pi\left(\mathrm{Yi}_{\mathrm{i}}+1\right) \sigma_{\mathrm{n}}^{2}}}+\pi+\frac{\mathrm{a} \lambda}{2\left(\mathrm{Y}_{\mathrm{i}}+1\right) \sigma_{n}^{2}}\right]$

b) When $z>0.5$

$r_{1} \cdot\left(t_{g i} w\right)^{4}+r_{2} \cdot\left(t_{g i} w\right)^{\mathrm{a}}+r_{\mathrm{a}} \cdot\left(t_{g i} w\right)^{2}+r_{4} \cdot\left(t_{g i} w\right)+r_{5}=0$

Where, the values of ${ }^{r_{1-5}}$ are constant. It can be calculated in tern of the constant $\mathrm{R}$ as follow:

$$
\begin{gathered}
R=2\left(\gamma_{i}+1\right) \sigma_{n 1}^{2} \\
r_{1}=4 R^{4} \\
r_{2}=15 R^{4} \\
r_{1}=2 R^{2}\left(8 R^{2}-4 \lambda^{2}-\lambda R\right) \\
r_{4}=17 \lambda^{2} R^{2} \\
r_{5}=4 \lambda^{4}
\end{gathered}
$$

c) When $\mathrm{z}<0.5$

$$
\left(t_{g i} w\right)^{a}+r_{1} \cdot\left(t_{g i} w\right)^{2}+r_{2}\left(t_{g i} w\right)+r_{a}=0
$$

Where, the values of $r_{1-a}$ are constant.

$$
\begin{gathered}
r_{1}=\frac{2\left(\gamma_{i}+1\right) \sigma_{n}^{2}-\lambda}{2\left(\gamma_{i}+1\right) \sigma_{n}^{2}} \\
r_{2}=-\frac{\lambda^{2}+6 \lambda\left(\gamma_{i}+1\right) \sigma_{n}^{2}}{\left(2\left(\gamma_{i}+1\right) \sigma_{n}^{2}\right)^{2}} \\
r_{\mathrm{a}}=\left(\frac{\lambda}{2\left(\gamma_{\mathrm{i}}+1\right) \sigma_{n}^{2}}\right)^{\mathrm{a}}
\end{gathered}
$$

\section{THE PARTICIPATE SENSORS SELECTION SCHEME}

The process of selecting the set of participate sensors according to each cooperative sensing process will present in this section relating to the proposed scheme selects ${ }^{\mathcal{N}}$. The goal behind this kind of selection is to satisfy, as possible, the maximum product of the utilization and the remaining power for each sensor node. However, the utilization $\left(U_{i}\right)$ can be formed for each secondary user, which is driven for ${ }^{\mathcal{N}}$ cooperative sensing nodes with the sensing time $\left(T_{S N i}\right)$ in previous work [4], as: 
$U_{i}\left(T_{W N i}, \hat{P}_{f i}\right)=\left(1-\frac{T_{M r i}}{T}\right)\left(1-\hat{P}_{f i}\right)$

Assuming that the total sensor's energy is equal to the sensing energy at the beginning of the operation $\left(\varepsilon_{T i}\right)$. Thus, $E_{g i}=e_{g} \cdot t_{g i}$ is the energy used for the new sensing, and $\left(\varepsilon_{v i}\right)$ is the reaming energy at sensor $i$ at the decision time. Accordingly, the difference between the energy available in the beginning and the total energy used for sensing represents the reaming energy $\left(\varepsilon_{r i}\right)$.

$\varepsilon_{r i}=\varepsilon_{T i}-E_{g i}$

The cost function per each sensor $\left(C F_{2 i}\right)$ determines by multiplying the utilization with reaming energy at sensor ${ }^{i}$, Therefore, the cost function can be recalled as:

$C F_{2 \mathrm{i}}=U_{\mathrm{i}}, \varepsilon_{\text {ri }}$

Substituting 17 and 18 in 19.

$$
C_{2 \mathrm{i}}=\left(1-\frac{T_{M i \mathrm{i}}}{T}\right)\left(1-\hat{P}_{f \mathrm{i}}\right)\left(\varepsilon_{T \mathrm{i}}-E_{g \mathrm{i}}\right)
$$

Depending on the above equation (20), the cost function for all sensors will be available to calculate. Subsequently, to have the highest cost function, set $\xi_{i}=1_{\text {for }}$ the $\mathrm{N}$ selected sensors under the limit of $Q_{d} \geq \widehat{Q_{d}}$, in order to satisfy the sensing performance, and $\sum i \xi_{i}=\mathcal{N}$. Where $\widehat{Q_{d}}$ is the target probability of detection.

Lastly, Assuming that $Q_{m}$ refers to the missing detection of the sensors, which is completely dependent on the false detection ${ }^{Q_{f}}$, where: $Q_{m}=1-Q_{f}$. Then, defiantly, the summation of the false and the missing detections presents the total detection error $\left(d e_{T}\right)$, as follow:

$d e_{T}=Q_{f}+Q_{m}$

\section{RESULTS AND DISCUSSION}

Depending on the mathematical model presented in previous two sections, this section described numerical calculation to validate the performance of the proposed scheme. While the final results of the simulation program, which is performed by using Matlab 2018b, compared with Mean absolute error (MAE) algorithm presented by [11], in order to prove the effectiveness of the proposed scheme. The main parameters that utilized to run the simulation program are listed in Table I.

Table I. The Main Simulation Parameters

\begin{tabular}{|c|c|c|c|}
\hline Name & Symbol & Value & Unit \\
\hline No. of battery powered constrained sensors & $\mathrm{I}$ & 60 & ------- \\
\hline sensing energy & $e_{g}$ & 0.05 & $\mathrm{~J}$ \\
\hline Independent distributed time & $T_{\text {on }}$ & 1 & $\mathrm{Sec}$ \\
\hline Identically distributed time & $T_{\text {off }}$ & 2 & $\mathrm{Sec}$ \\
\hline Target probability of detection & $\widetilde{Q}_{d \dot{d}}$ & 0.9 & ------- \\
\hline Total sensing energy & $\varepsilon_{T i}$ & 1000 & $\mathrm{~J}$ \\
\hline Bandwidth & $W$ & 6 & $\mathrm{MHz}$ \\
\hline Ground noise & $\sigma_{\mathrm{n}}$ & -5 & ------- \\
\hline
\end{tabular}


Firstly, the total error, presented in equation 21, was determined against multi-values of energy threshold $\left({ }^{\lambda}\right)$ in $\mathrm{dB}$ unit, in order to determine the perfect threshold $\left(^{\lambda}\right)$ that can be used in the rest of calculations. Indeed, six various thresholds $(\lambda)$ was used in different values of SNR, $-30,-20,-10,0,10,20 \mathrm{~dB}$, respectively, as shown in Figure 1. The results clearly identify that the minimum range of the total error has appeared during the range $7.5 d B \leq \lambda \leq 10 d B$. Based on this conclusion, the best threshold, $\lambda=10 \mathrm{~dB}$, will be taken with the current simulation.

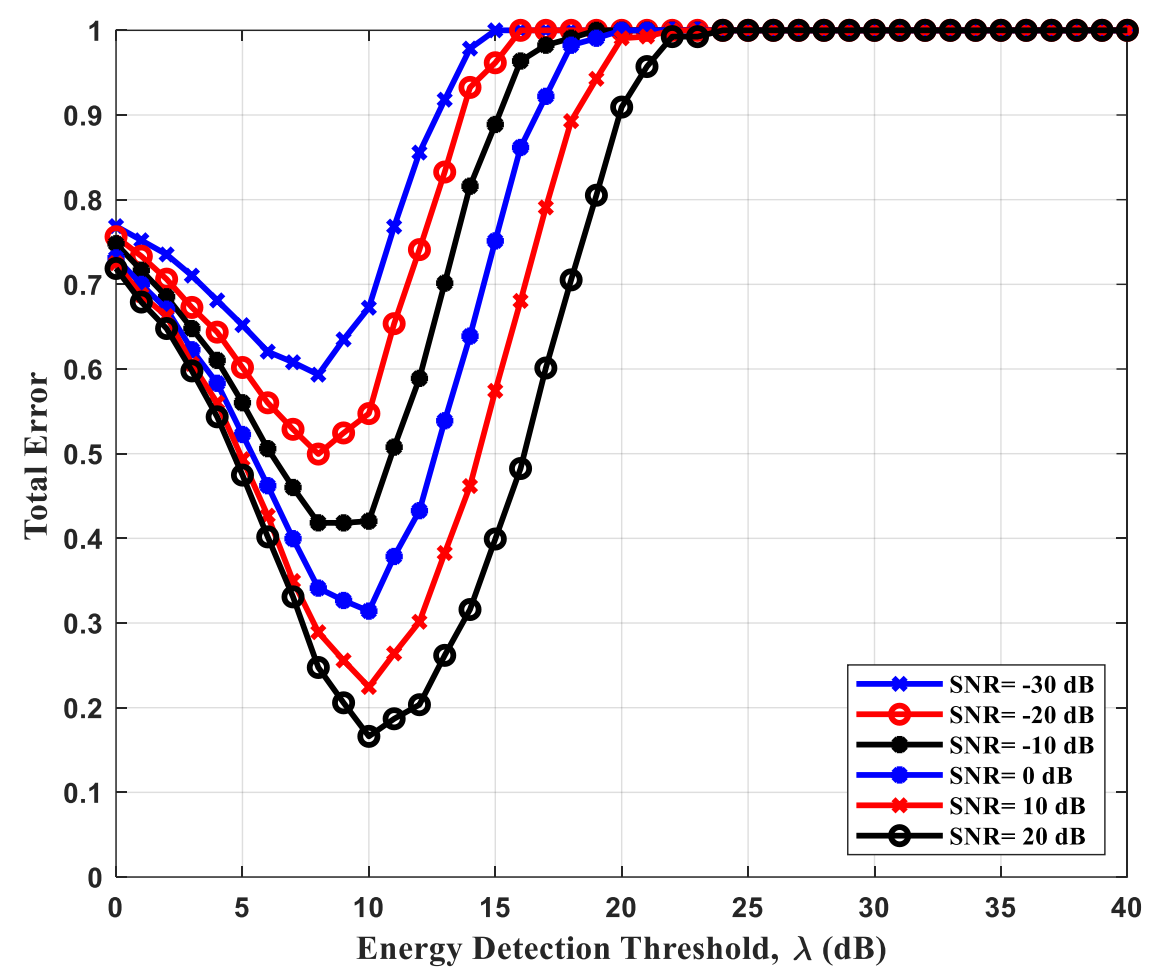

Fig. 1 The behavior of the total error at several values of SNR

On the other hand, Figure 2 shows the behaviour of the proposed scheme against different values of SNR for each sensor. Where the horizontal axis refers to the average SNR, while vertical axes of the subplots a-f indicate to the average sensing power, utilization, threshold for cooperative false alarm probability, a threshold for cooperative detection probability, total error, and lifetime, respectively. Consequently, the results of the proposed scheme were presented in a blue continuous line and the outputs of the mean absolute error (MAE) algorithm were plotted in a black intermittent line.

In general, the proposed scheme leads to reduce the average sensing power for each individual sensor, as shown in Figure 2.a. Indeed, the utilizing average power per each sensor is going to be lower when the average SNR increases, i.e, the usage sensing time of each sensor during the sensing operation becomes relatively shorter. At the same operation time, the utilization of the proposed model shows increasing behaviour when the average SNR increases, see Figure 2.b. Therefore, increasing the utilization leads to highly augment the usage efficiency of the available spectrum by secondary users. 

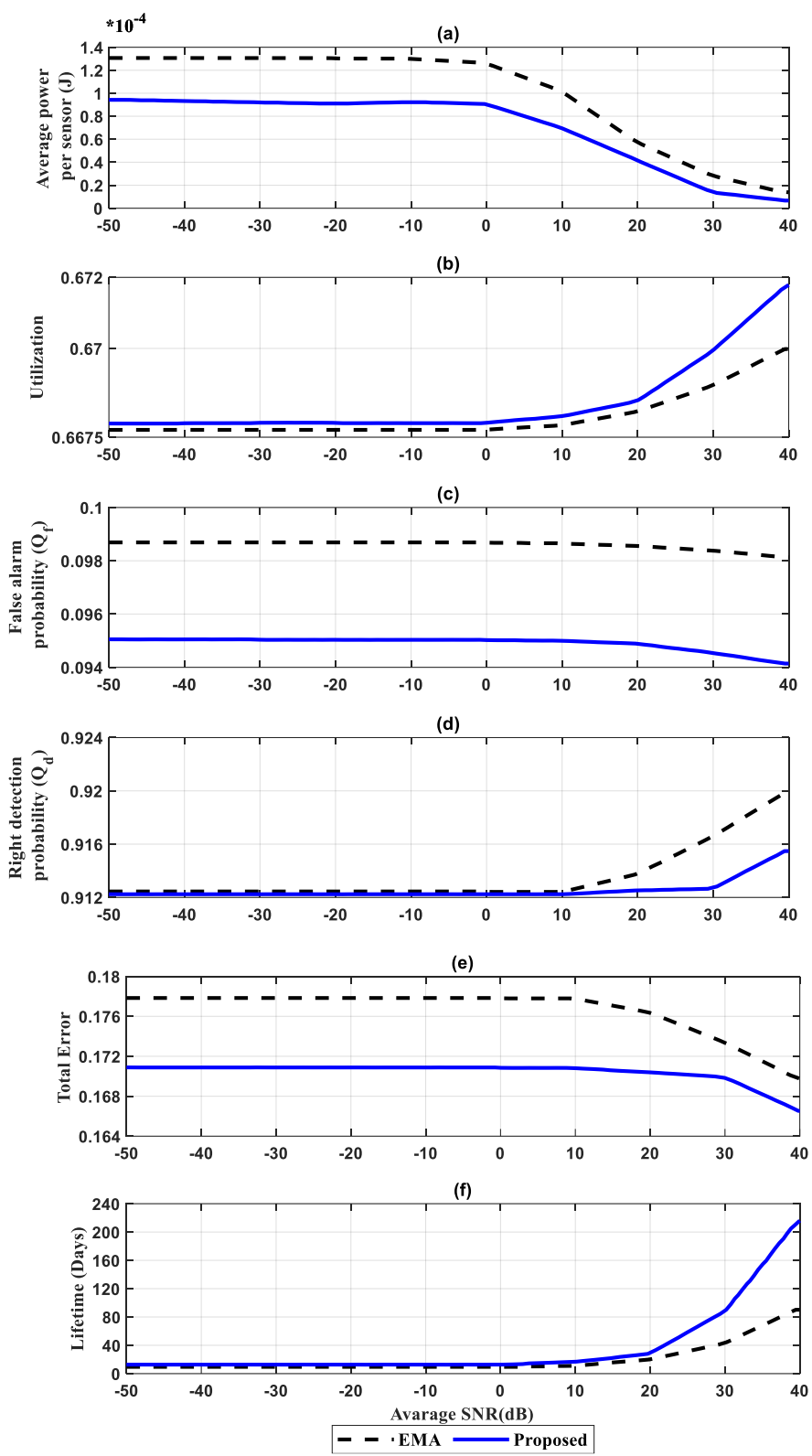

Fig. 2 The behavior of the proposed scheme against different values of SNR: a) average sensing power, b) utilization, c) threshold for cooperative false alarm probability, d) threshold for cooperative detection probability, e) total error, and f) lifetime.

Figures 2.c and 2.d illustrate the detection sensing performance based on the proposed scheme in the mean of the cooperative false alarm and cooperative detection probabilities. The performance of the two probabilities was pretty improved for all values of average SNR comparing with the existing solving of the MAE algorithm. Accordingly, the improvement in the detection performance of the cooperative false alarm probability and the cooperative detection probability definitely leads to clearly reduce the detection total error per each sensor against different values of SNR, as described in Figure 2.e. Thus, the reduction that occurred on the average sensing power and the total error per each sensor leads to exceptionally improve the lifetime of the sensing node, in specific, and the lifetime of the network, in general, see Figure 2.f.

Finally, the main contribution of this work has described in the Figure 3 . The figure shows the percentage increase in the lifetime per the sensing node against different values 
of SNR. It is clear to note that the lifetime's percentage has proportionally raised with the increasing of the average SNR and its reach to its maximum value (134 days) at the 40 dB SNR. Accordingly, it can conclude that, based on the proposed scheme, the percentage of the sensing lifetime improves with increased SNR.

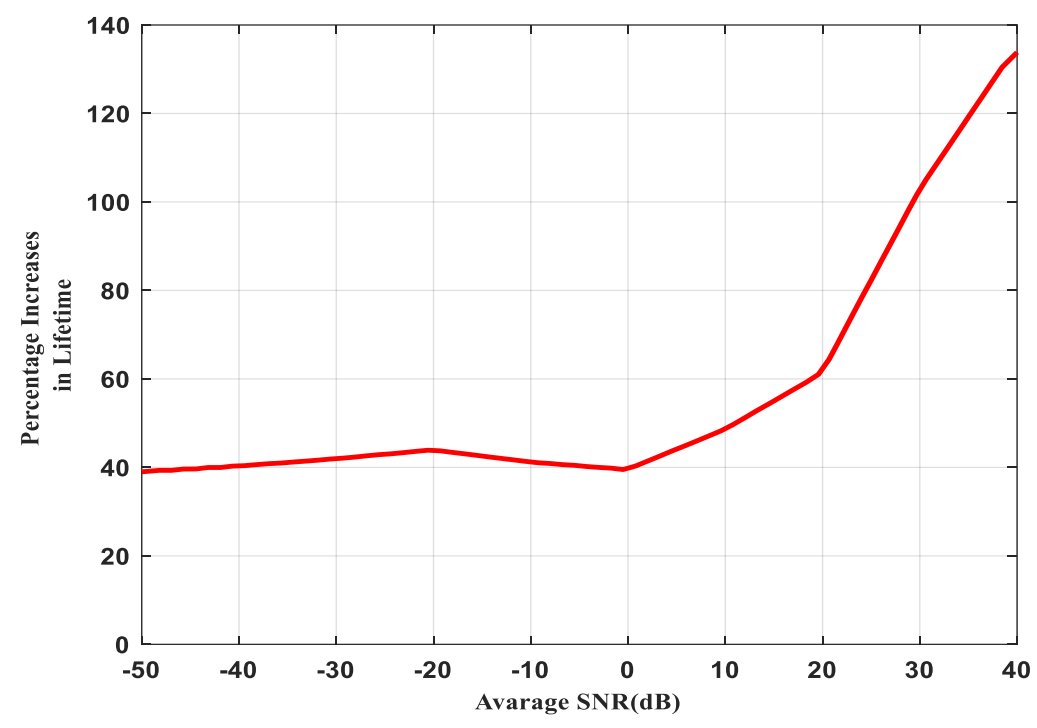

Fig. 3 The lifetime's percentage against different values of SNR.

\section{CONCLUSION}

The minimization of the total energy consumed by a group of power-constraint sensors for cooperative spectrum sensing in sensor-aided cognitive radio networks was studied in this work. A new cooperative spectrum sensing scheme was driven based on the average sensing energy of each sensor inside the network. The simulation results proved that the proposed scheme reduced the average sensing energy and the total detection error for each sensor. Thus, the lifetime of the CR will increase by minimizing the sensing energy for each sensor and increases the utilization of the available spectrum by the secondary users. In conclusion, the results of the proposed scheme show extremely suitable detection performance of the cognitive network.

The question of how to efficiently coordinate and select the sensors that experience the highest SNR and that are well separated from each other in order to avoid correlation shadowing in cooperative spectrum sensing is addressed as the future direction of this paper. In addition, examination of the performance of the proposed scheme at different applying voting role and applying double threshold are highly recommend to investigate in the future works.

\section{REFERENCES}

[1] Mitola III, Joseph. "Cognitive radio for flexible mobile multimedia communications." Mobile Networks and Applications 6.5 (2001): 435-441.

[2] Akyildiz, Ian F., Won -Yeol Lee, Mehmet C. Vuran, and Shantiedv Mohanty. "NeXt generation/dynamic spectrum access/cognitive radio wireless networks: A survey." Computer networks 50.13 (2006): 2127-2159.

[3] Federal Communications Commission, Spectrum policy task force report, Tech. Rep. ET Docket No. 02-155, Editor. 2002.

[4] Sunghwan, Bae and Kim Hongseok. "Unlimited cooperative sensing with energy detection for cognitive radio." Journal of Communications and Networks 16.2 (2014): 172-182. 
[5] IEEE, Ieee p802.22/d1.0 draft standard for wireless regional area networks part 22: Cognitive wireless ran medium access control (mac) and physical layer (phy) specifications: Policies and procedures for operation in the tv bands. 2008.

[6] Zhang, Wei., Ranjan K Mallik, and Khaled Ben Letaief. "Cooperative spectrum sensing optimization in cognitive radio networks." International Conference on Communications, 19, May 2008. IEEE, pp. 3411-3415.

[7] Mishra, Shridhar Mubaraq, Anant Sahai and Robert W Brodersen. "Cooperative sensing among cognitive radios." in Icc. 2006. Citeseer.

[8] Shinde, S.C. and A.N. Jadhav. "Centralized cooperative spectrum sensing with energy detecion in cognitive radio and optimization." International Conference on Recent Trends in Electronics, Information \& Communication Technology (RTEICT). Bangalore, India, 2016. IEEE, pp. 1002-1006

[9] Gupta, Manish S, Gaurav D. Verma and Rahul Kumar Dubey. "Cooperative spectrum sensing for cognitive radio based on adaptive threshold." Second International Conference on Computational Intelligence \& Communication Technology (CICT). Ghaziabad, India, 2016. IEEE, pp. 444-448

[10] Yang, G., J. Wang, J. Luo, O. Y Wen, H. Li, O. Li and S. Li. "Cooperative spectrum sensing in heterogeneous cognitive radio networks based on normalized energy detection." IEEE Transactions on vehicular technology 65.3 (2015): 1452-1463

[11] Pham, H.N., Y. Zhang, P. E. Engelstad, T. Skeie and F. Eliassen. "Energy minimization approach for optimal cooperative spectrum sensing in sensor-aided cognitive radio networks." The 5th Annual ICST Wireless Internet Conference (WICON). Singapore, 2010. IEEE.

[12] Naveen, Kanne and Yedunuri Shekar. "Cooperative Spectrum Sensing Parameter Optimization Algorithm in Cognitive Radio System.” International Journal of Engineering and Advanced Technology 8.4 (2019): 1166-1169

[13] Lee, Won -Yeol and Ian F. Akyildiz. "Optimal spectrum sensing framework for cognitive radio networks." IEEE Transactions on wireless communications 7.10 (2008): 3845-3857.

[14] Maleki, Sina, Ashish Pandharipande and Geert Leus. "Energy-efficient distributed spectrum sensing for cognitive sensor networks." IEEE sensors journal 11.3 (2011): 565-573.

[15] Jenifer, Angel J., R. Gokul and Punal M Arabi. "Maximization the lifetime of cognitive sensor network using sub fusion centers." ARPN Journal of Engineering and Applied Sciences 10.4 (2015): 1774-1777.

[16] Li, Peng, Zhikui Chen, Laurence Yang T., Qingchen Zhang and M. Jamal Deen."Deep convolutional computation model for feature learning on big data in Internet of Things." IEEE Transactions on Industrial Informatics 14.2 (2018): 790-798.

[17] Li, Peng., Zhikui Chen, Laurence Yang T., Liang Zhao, Qingchen Zhang, "A privacy-preserving highorder neuro-fuzzy c-means algorithm with cloud computing." Neurocomputing 256 (2017): 82-89.

[18] Chen, Yangyi, Shaojing Su, Huiwen Yin, Xiaojun Guo, Zhen Zuo, Junyu Wei and Liyin Zhang. "Optimized Non-Cooperative Spectrum Sensing Algorithm in Cognitive Wireless Sensor Networks." Sensors 19.9 (2019): 2174

[19] Zhang, Xue, Xiaozhu Liu, Hooman Samani and Brian Jalaian. "Cooperative Spectrum Sensing in Cognitive Wireless Sensor Networks." International Journal of Distributed Sensor Networks 11.8 (2015): 1-15

[20] Gradshteyn, I. and I. Ryzhik. "Table of Integrals, Series and Products 7th edn, ed A Jeffrey and D Zwillinger" New York: Academic. 2007.

[21] Maharjan, Sabita, Chau Yuen, Yong Chew Huat, Yan Zhang and Stein Gjessing."Distributed spectrum sensing for cognitive radio networks with heterogeneous traffic." the 3rd International Symposium on Applied Sciences in Biomedical and Communication Technologies Rome, Italy. 7-10 Nov. 2010. IEEE.

[22] Varshney, P. K. "Distributed Detection and Data Fusion, ser. Signal processing and data fusion, C. S. Burrus." Ed. New York: Springer, 1997.

[23] Pham, H.N., Y. Zhang, P. E. Engelstad, T. Skeie and F. Eliassen. "Optimal cooperative spectrum sensing in cognitive sensor networks." in Proceedings of the 2009 International Conference on Wireless Communications and Mobile Computing: Connecting the World Wirelessly. 2009. ACM. 